\title{
Investigating Predictors of Online Vigilance among University Students
}

\author{
Daniel B. le Roux \\ Corresponding author: dbleroux@sun.ac.za \\ Department of Information Science, Stellenbosch University, South Africa \\ Orcid ID: https://orcid.org/0000-0001-9682-0377 \\ Douglas A .Parry \\ Department of Information Science, Stellenbosch University, South Africa \\ Orcid ID: https://orcid.org/ 0000-0002-6443-3425
}

This is the Author Accepted Manuscript (AAM) of the article published in the Journal of Information Technology \& People at https://doi.org/10.1108/ITP-04-2020-0226

\begin{abstract}
Purpose

Online vigilance is a novel construct which describes individual differences in users' cognitive orientation to online connectedness, their attention to and integration of online-related cues and stimuli, and their prioritisation of online communication. Its proponents argue that it is acquired through the processes of instrumental and attentional training that underlie media use behaviours. The purpose of the present study is to investigate the role of three personal characteristics (emotional intelligence, rumination and identity distress) as predictors of online vigilance in addition to media use behaviour.

\section{Design/methodology/approach}

We adopted an exploratory frame and followed a survey-methodology to collect data among a sample of university students $(n=812)$. The resulting data was analysed through a hierarchical multiple regression in which four models were considered.
\end{abstract}

\section{Findings}


Our findings indicate that while media use behaviours (daily smartphone use, social media use, messaging, video watching and media multitasking) predict online vigilance, their combined effect is weak. However, when considering these behaviours in combination with trait rumination and identity distress, a moderate effect is observable.

\section{Research limitations/implications}

While our findings do not permit causal inference, it suggests that two personal characteristics, trait rumination and identity distress, play an important role in determining an individual's tendency or ability to psychologically disconnect from their online spheres. This provides an initial step towards the theorisation of online vigilance and the identification of individuals who may be at risk of acquiring it.

\section{Originality/value}

Online vigilance is a novel construct which has only been investigated in a small number of studies. However, its emphasis on psychological connectedness presents a unique and important development in the context of permanently online, permanently connected living. The present study is the first to explore its association with personal characteristics.

\section{Keywords}

Emotional Intelligence, Identity Distress, Media Multitasking, Online Vigilance, Personal characteristics, POPC, Rumination, Social media

\section{Introduction}

The construct of Online Vigilance and its associated assessment instrument (The Online Vigilance Scale; Reinecke et al., 2018), provides a novel lens through which researchers can investigate the potential effects of permanently online, permanently connected living on attentional distribution strategies and well-being. Online vigilance refers to individual differences in three dimensions of users' cognitive orientation to online connectedness. The first dimension, salience, concerns the degree to which people dedicate thoughts to their online 
spheres. The second dimension, monitoring, refers to people's tendency to enter their online spheres on a regular basis, and the third dimension, reactibility, refers to people's prioritisation of events and cues from their online spheres over demands from their offline environments. Individuals that "are high in online vigilance will think more often and more intensively about their personal online sphere even when they are not using their mobile devices" (Reinecke et al., 2018 , p. 2). The contruct's proponents argue that online vigilance is acquired through processes of instrumental and attentional learning that occur during interactions with the affordances of online platforms. Such platforms provide the ideal conditions for these learning processes because they "represent a reliable source of intrinsic need satisfaction" and offer a diverse range of gratifications (Reinecke et al., 2018, p. 3). Importantly, however, the authors distinguish online vigilance from Internet addition which, they argue, involves extreme or pathological forms of salience. Online vigilance, by contrast, represents "a much more mundane form of involvement with the online environment that does not necessarily impair individual functioning and mental health" (Reinecke et al., 2018, p. 7).

While a majority of studies investigating effects associated with chronic media use target direct relationships between indications of media use and well-being (or other) outcomes, the notion of online vigilance proposes that these effects are mediated by a particular cognitive state (i.e., online vigilance). This represents an important development in Media Psychology research for two primary reasons. Firstly, it accepts the principle that there exists variance in the degree to which media use behaviours cultivate cognitive preoccupation with online connectedness and content. In this regard Reinecke et al. (2018) argue that the affordances of online communication "do not possess the same subjective value for all users" (p. 8), alluding to the proposition that users with the same media use patterns can develop different levels of online vigilance. Secondly, when considering media effects, the construct directs attention to the cognitive state produced by chronic media use rather than the media use behaviours themselves. This distinction between the behavioural pattern and the cognitive orientation provides a basis for the investigation of the role that personal characteristics play in the development of online vigilance (Reinecke et al., 2018). It has been proposed that, among other factors, perceived social pressure, self-esteem, and need satisfaction may account for individual differences in online vigilance (Reinecke et al., 2018). 
While few studies have investigated the effects of online vigilance for subjective well-being, extant evidence suggests that it is positively associated with mind-wandering and fear of missing out, and negatively associated with affective well-being and mindfulness, all among samples primarily consisting of younger individuals (Johannes et al., 2018; Schneider and Hitzfeld, 2019). These associations may point to potential causal factors but, at present, no research adopting either experimental or longitudinal techniques has been conducted to identify factors producing, mediating, or moderating tendencies towards online vigilance in either student or adult populations. Despite this, across these studies, a pattern is discernible. Associations, albeit weak to moderate, between the cognitive orientation to and frequent monitoring of online communication are, primarily, related to a tendency to engage in task-unrelated thoughts and a fear of missing out ("a pervasive apprehension that others might be having rewarding experiences from which one is absent”; Przybylski et al., 2013).

\section{Online Vigilance and Media Use}

Little is currently known about associations between online vigilance and particular forms of media use behaviour. Reinecke et al., (2018, p.14) report "strong connections" between Internet Multitasking, conceptualised as internet use during other media use, work or conversations, and online vigilance and interpret this finding to indicate that users with high levels of online vigilance are constantly willing to "make room" for Internet use (Reinecke et al., 2018, p.16). This supports the notion of online vigilance as a "strong and permanent occupation with and motivational prioritization of Internet use irrespective of primary activities and the demands of the offline context" (Reinecke et al., 2018, p. 16). This argument corresponds with a growing body of evidence about media multitasking in general (i.e., the simultaneous use of two or more media; Baumgartner et al., 2017), which suggests that media users often experience goal interference due to their permanent connectedness. Media multitasking has been shown to be a broadly adopted behavioural pattern among the current generation of university students (le Roux and Parry, 2019a) and has been associated with reduced performance in various domains of cognitive control (sustained attention, inhibition, filtering; Wiradhany and Nieuwenstein, 2017). However, its interaction with online vigilance is currently unknown. 


\section{Online Vigilance and Personal Characteristics}

In addition to the limited evidence of association between media use behaviours and online vigilance, its relationships with personal characteristics are yet to receive research attention. A wide range of personal characteristics have been studied in relation to online media use behaviours. The present study considers three of these - emotional intelligence, rumination and identity distress. These characteristics were chosen on the basis of their reported associations with theoretical constructs that are adjacent to or congruent with one or more of the dimensions of online vigilance (e.g., media multitasking and problematic social media use; Dempsey et al., 2019; Hornung et al., 2018; Kircaburun, 2019; Süral et al., 2019; Ward, 2017; Yang et al., 2018).

Trait emotional intelligence represents a constellation of emotional self-perceptions located at the lower levels of personality hierarchies (Petrides et al., 2007). The construct comprises four multifaceted components: emotionality, sociability, self-control, and well-being, as well as two independent facets (adaptability and self-motivation). In contrast to other models of emotional intelligence, trait emotional intelligence is considered to be representative of personality traits, as opposed to mental abilities (Petrides, 2009). Individuals that report high trait emotional intelligence are generally able to control their emotions, perceive their own and others' emotions accurately, have good social skills, and adopt a positive outlook on life (Petrides, 2009; Petrides et al., 2007). There is, as of yet, no reported studies presenting empirical evidence of the interplay between trait emotional intelligence and online vigilance. However, previous studies of trait emotional intelligence, suggest that it may act as a protective factor against various health and behavioural problems for adolescents (Kircaburun et al., 2019). Among school students it was found that those with higher trait emotional intelligence and mindfulness displayed reduced rumination and engaged in lower levels of problematic social media use (PSMU) (Kircaburun et al., 2019). In a study of PSMU among university students, both direct and indirect relationships between trait emotional intelligence and PSMU were found via two social media use motives: expressing or presenting a more popular self and passing time (Süral et al., 2019). The authors suggest that, for individuals with low trait emotional intelligence, PSMU presents as a coping 
strategy adopted to deal with life challenges. This finding is supported by a qualitative study of student media use behaviours which found that media use is often undertaken by students in an attempt to stabilise mood (le Roux and Parry, 2019a). A recent study, building upon earlier evidence of stress experienced as a result of social media use, suggests that individuals higher in emotional intelligence are better equipped to cope with this form of stress due to lower fear of judgement or rejection (Hornung et al., 2018). The study hypothesised, on this basis, that high emotional intelligence will correlate positively with Facebook use. Support for this hypothesis was found when considering younger users, but the opposite was found to be the case for users older than 25. The authors comment that, while younger users may have developed emotional intelligence along with social media use, older users may be "alienated by the frequent superficial relationships on Facebook" (Hornung et al., 2018, p. 8).

Conceptualisations of rumination generally fall into one of two categories. The first category, which is most frequently adopted by researchers (Brinker and Dozois, 2009), frames the construct as a coping mechanism generally associated with depressed mood. During depressive rumination thoughts and behaviours focus the depressed individual's attention on past mistakes and failures (McLaughlin et al., 2007), as well as their symptoms and the causes and consequences of those symptoms (Butler and Nolen-Hoeksma, 1994). Such rumination is considered a maladaptive emotion regulation strategy adopted more frequently by females than males (Nolen-Hoeksema, 2001). However, rumination has also been conceptualised as a dispositional ruminative thought style independent of a particular content domain (Brinker and Dozois, 2009). This second category includes but does not limit the construct to depressive rumination, enabling the exploration of rumination in alternative content domains. The role of depressive-oriented rumination as a mediator between trait emotional intelligence and problematic online behaviours among adolescents has been investigated (Kircaburun, 2019). Findings suggest that ruminating adolescents attempt to avoid negative thoughts and images by excessively engaging in online activities and this, in turn, can become problematic. This aligns with findings indicating a positive correlation between rumination and problematic Facebook use which suggests that rumination about offline relationships may drive excessive social media use with the aim of satisfying or relieving such rumination and alleviating negative mood (Dempsey et al., 2019). The relationship between social comparison on social media and rumination among 
university students has also been investigated (Yang et al., 2018). The study distinguished between social comparison of ability during which individuals compare their performance or achievement to that of others, and social comparison of opinion during which comparison is based on differences in attitudes, beliefs, and values. A positive correlation between social comparison of ability on social media and rumination was found, but no association between the social comparison of opinion and rumination. In addition, a positive correlation between social comparison of ability and identity stress was found.

Identity distress describes difficulties experienced in the process of identity development. While it is broadly accepted that a degree of distress is generally experienced by all individuals during identity development, the level of distress can, in extreme cases, become overwhelming. This may disrupt the development processes and impair normal functioning (Berman et al., 2004). While identity distress is not recognised as a disorder in the current Diagnostic and Statistical Manual of Mental Disorders (DSM-V), some of its characteristics are recognised as key components in various personality and dissociative disorders (Yang et al., 2018). Considering its potential interaction with online vigilance, Yang et al., (2018) note that online media expose adolescents to an increasingly wide range of choices concerning key identity factors like value systems, worldview, religion and sexual orientation. Ward (2017, p. 34), accordingly, argues that adolescents' "online and offline lives have become inextricably intertwined and that while social media can provide valuable opportunities for adolescent identity exploration, it can also have a significant impact on identity exploration [...] and may be placing adolescents at risk". She further posits, given the primacy of achieving identity coherence during adolescence, that "it can be hypothesised that anxious individuals experiencing low self-concept clarity and identity confusion may spend more time on social media experimenting with their identity in an attempt to manage their psychological distress" (p. 35). These arguments suggest that identity distress may increase adolescents' cognitive orientation towards online media, as well as their monitoring of and responding to online content and communication.

\section{Research Questions}


Given the relative novelty of the domain, and the absence of an applicable theoretical framework, the present study adopted an exploratory frame and, building on the arguments presented in this section, set out to investigate potential predictors of online vigilance in a systematic manner. This is done, firstly, by determining the association between online vigilance and daily smartphone use, the use of different media types, and media multitasking. Thereafter, we extend this analysis by determining the degree to which the three personal characteristics identified in the literature review provide additional explanation for variance in online vigilance among students. The following research questions were formulated to structure these objectives:

RQ1: How much variability in online vigilance does daily smartphone use account for? RQ2: How much variability in online vigilance does social media use, messaging and video watching account for over and above that accounted for by daily smartphone use? RQ3: How much variability in online vigilance does media multitasking account for over and above that accounted for by use?

RQ4: How much variability in online vigilance do emotional intelligence, rumination and identity distress account for over and above that accounted for by media use and multitasking?

\section{Method}

Participants

We targeted a student sample and recruited respondents through an email invitation sent to all undergraduate students at a large residential university in South Africa. In total, 1337 responses to the survey were received, from which 433 incomplete and 92 ineligible (we set a lower bound for inclusion at the 5th percentile of completion speed and an upper bound at the 95th percentile) responses were removed, leaving a final sample of $n=812$ undergraduate students $(62.68 \%$ female).

Instruments 
Daily smartphone use was measured using a single item with a Likert scale ranging from 1 (Less than 30 minutes) to 9 (More than 4 hours).

Online Vigilance was assessed with the Online Vigilance Scale (Reinecke et al., 2018). Consisting of three subscales — one for each of the three dimensions of online vigilance (salience, monitoring, and reactivity) - 12 items are presented (four for each subscale). Responses are provided through Likert scales ranging from 1 (Does not apply at all) to 5 (Fully applies). For the salience subscale items focus on cognitive orientation (e.g., "I constantly monitor what is happening online" and "I have a hard time disengaging mentally from online content"). For the monitoring subscale items focus on the chronic monitoring of online content and messages (e.g., "I constantly monitor what is happening online" and "I always keep an eye on what is happening online at the moment"). For the reacting subscale items focus on immediate responsiveness to online messages (e.g., "When I receive an online message I immediately give it my full attention" and "When I receive an online message it triggers an impulse in me to check it right away"). For each subscale the relevant items were averaged (salience: $M=2.18, S D=0.86$; monitoring: $M=2.33, S D=1.04$; reacting: $M=2.97, S D=$ 1.01). To form an overall scale for online vigilance the responses for each dimension were averaged.

To assess media multitasking we used the Media Multitasking Index-Short (MMI-S; Baumgartner et al., 2017), which considers multitasking across three primary activities (watching $\mathrm{TV}$, using social media, and sending messages via phone or computer) and four secondary activities (the three primary activities and listening to music). We amended the 'watching TV' item to include a broader range of video-related media and represented it as 'video watching'. For each of the primary activities respondents indicate on a Likert scale from 1 (not at all) to 6 (3 hours or more) their use-duration on an average day. For each of the secondary items participants indicate, on a Likert scale from 1 (never) to 4 (very often), how often they engage simultaneously with each of the primary items. To calculate the MMI-S these values were combined using the conventional formula (Ophir et al., 2009). 
The Ruminative Thought Styles Questionnaire (RTSQ; Brinker and Dozois, 2009) was used to assess trait rumination. Through Likert scales ranging from 1 (Not at all) to 7 (Very well), respondents indicate how well each of 20-items, including "I find myself reliving events again and again" and "I find that some thoughts come to mind over and over throughout the day", describe them. Responses for each item are summed, with a higher value representing greater trait rumination.

Trait emotional intelligence was assessed with the Trait Emotional Intelligence QuestionnaireShort Form (TEIQue-SF; Petrides, 2009). Responses are provided to 30-items, including "I usually find it difficult to regulate my emotions" and "On the whole, I'm a highly motivated person" through Likert scales ranging from 1 (Completely disagree) to 7 (Completely agree). Outcomes are averaged with a higher value representing greater trait emotional intelligence.

Identity Distress was assessed with the Identity Distress Survey (IDS; Berman et al., 2004), asking respondents to indicate the degree to which they have recently been upset, distressed or worried about seven identity-related issues (e.g., career choice, friendships, sexual orientation and religion). Likert scales ranging from 1 (not at all) to 5 (very severely) were used for all items. Outcomes are averaged with a higher value representing greater identity distress.

\section{Procedure}

All survey materials are in the public domain and are available through the Open Science Framework (https://osf.io/k862z/?view_only=1ac17e9c949844f6981fbef2d5f30157). The survey was captured on a web-based platform and invitations were distributed via email to all undergraduate students at the relevant institution. Data collection took place over a two-week period, with analysis only occurring upon completion.

\section{Ethical Considerations}

Ethical clearance for the study and permission to distribute invitations to complete the survey was obtained from the relevant institutional bodies (Research Ethics Committee: Social 
Behavioural and Education Research; REC:SBE), with the study being deemed 'low risk'. All participants indicated informed consent to the study purpose, methods, and analysis and reporting approach, before voluntarily completing the survey.

\section{Data Analysis}

All data analyses were conducted using the $R$ statistical programming language (v. 3.5.1). The analysis script is available on the Open Science Framework (https://osf.io/53uvj/?view_only=d17fc1c8c7a145a0b4165f09c55244f6). After producing the relevant scales, to address possible multicollinearity, the analysis first involved assessing zeroorder bivariate correlations among all study variables. In addition, we considered internal consistency with Cronbach's $\alpha$. To address our research questions we conducted a hierarchical multiple regression analysis, involving four steps (one for each RQ), predicting online vigilance as the dependent variable.

\section{Findings}

Descriptive statistics and zero-order correlations for all the study variables are displayed in Table 1. With the exception of the relationships between emotional intelligence and messaging $(p=0.35)$, as well as emotional intelligence and social media $(p=0.06)$, small to moderate significant correlations were found among all the study variables. 
Table 1: Descriptive statistics and zero-order correlations among study variables.

\begin{tabular}{|c|c|c|c|c|c|c|c|c|c|c|c|c|}
\hline & Variable & M & SD & $\alpha$ & 1 & 2 & 3 & 4 & 5 & 6 & 7 & 8 \\
\hline 1 & Daily smartphone use & 6.25 & 2.18 & & - & & & & & & & \\
\hline 2 & Messaging & 4.17 & 1.36 & & $0.61^{* * *}$ & - & & & & & & \\
\hline 3 & Video watching & 3.68 & 1.38 & & $0.42^{* * *}$ & $0.21^{* * *}$ & - & & & & & \\
\hline 4 & Social media & 3.84 & 1.43 & & $0.60^{* * *}$ & $0.57^{* * *}$ & $0.27^{* * *}$ & - & & & & \\
\hline 5 & Media multitasking & 1.52 & 0.67 & & $0.44^{* * *}$ & $0.46^{* * *}$ & $0.28^{* * *}$ & $0.54^{* * *}$ & - & & & \\
\hline 6 & Emotional intelligence & 4.85 & 0.76 & 0.87 & $-0.17^{* * *}$ & -0.03 & $-0.21^{* * *}$ & -0.07 & $-0.11^{* * *}$ & - & & \\
\hline 7 & Rumination & 90.38 & 22.15 & 0.93 & $0.20^{* * *}$ & $0.13^{* * *}$ & $0.17^{* * *}$ & $0.14^{* * *}$ & $0.24^{* * * *}$ & $-0.48^{* * *}$ & - & \\
\hline 8 & Identity distress & 2.27 & 0.71 & 0.76 & $0.23^{* * *}$ & $0.15^{* * *}$ & $0.14^{* * *}$ & $0.20^{* * *}$ & $0.26^{* * *}$ & $-0.40^{* * *}$ & $0.41^{* * *}$ & - \\
\hline 9 & Online vigilance & 2.49 & 0.79 & 0.90 & $0.28^{* * *}$ & $0.21^{* * *}$ & $0.09^{*}$ & $0.29^{* * *}$ & $0.32^{* * *}$ & $-0.20^{* * *}$ & $0.43^{* * *}$ & $0.33^{* * *}$ \\
\hline
\end{tabular}

Note: $\mathrm{N}=812 . \alpha$ represents Cronbach's alpha

$* p<.05 . * * p<.01 . * * * p<.001$.

The results of the hierarchical multiple regression analysis are presented in Table 2. The four models fitted in the analysis correspond to the four research questions posed. Results for Model 1 indicated that daily smartphone use predicted online vigilance $\left(R^{2}=0.076, F(1 ; 180)=66.91\right.$, $p<0.001)$. The second model fitted included the three media platforms/types used as additional independent variables and explained an additional $2.4 \%$ of the variance in online vigilance $\left(R^{2}=0.100, F(4 ; 807)=22.45, p<0.001\right)$. However, only daily smartphone use $(\beta=0.17, \mathrm{p}<0.001)$ and social media use $(\beta=0.19, \mathrm{p}<0.001)$ were significant predictors of the dependent variable. In the third model, which explained an additional $3.3 \%$ of variance in online vigilance, media multitasking $(\beta=0.17, \mathrm{p}<0.001)$ was added to the set of independent variables $\left(R^{2}=0.133\right.$, $F(5 ; 806)=24.88, p<0.001)$. Emotional intelligence, rumination and identity distress were added for the fourth model which explained an additional $14.3 \%$ of variance in online vigilance $\left(R^{2}=0.276, F(8 ; 803)=38.22, p<0.001\right)$. Of these three variables, rumination $(\beta=0.34, \mathrm{p}<0.001)$ was the strongest predictor, while identity distress was also significant $(\beta=0.15, \mathrm{p}<0.001)$. Interestingly, emotional intelligence was not a significant predictor of online vigilance when controlling for the other explanatory variables in the model. 
Table 2: Hierarchical multiple regression analysis predicting online vigilance.

\begin{tabular}{|c|c|c|c|c|c|c|c|c|c|c|c|c|c|c|c|c|}
\hline \multirow[t]{2}{*}{ Variable } & \multicolumn{4}{|c|}{ Model 1} & \multicolumn{4}{|c|}{ Model 2} & \multicolumn{4}{|c|}{ Model 3} & \multicolumn{4}{|c|}{ Model 4} \\
\hline & $B$ & SE & $\beta$ & $t$ & $B$ & SE & $\beta$ & $t$ & $B$ & SE & $\beta$ & $t$ & $B$ & $\mathrm{SE}$ & $\beta$ & $t$ \\
\hline Daily smartphone use & 0.10 & 0.01 & 0.28 & $8.18^{* * *}$ & 0.06 & 0.02 & 0.17 & $3.60^{* * * *}$ & 0.06 & 0.02 & 0.16 & $3.39^{* * *}$ & 0.04 & 0.02 & 0.11 & $2.51^{*}$ \\
\hline Messages & & & & & 0.01 & 0.03 & 0.01 & 0.24 & -0.02 & 0.03 & -0.03 & -0.71 & -0.01 & 0.02 & -0.02 & -0.49 \\
\hline Social media & & & & & 0.10 & 0.02 & 0.19 & $4.20^{* * * *}$ & 0.06 & 0.03 & 0.10 & $2.19^{*}$ & 0.06 & 0.02 & 0.11 & $2.62^{* *}$ \\
\hline Video & & & & & -0.02 & 0.02 & -0.04 & -0.99 & -0.04 & 0.02 & -0.06 & -1.74 & -0.05 & 0.02 & -0.1 & $-2.65^{* *}$ \\
\hline MMI & & & & & & & & & 0.27 & 0.05 & 0.23 & $5.59^{* * *}$ & 0.16 & 0.04 & 0.13 & $3.47^{* * *}$ \\
\hline Identity distress & & & & & & & & & & & & & 0.15 & 0.04 & 0.13 & $3.83^{* * * *}$ \\
\hline Emotional intelligence & & & & & & & & & & & & & 0.04 & 0.04 & 0.04 & 1.03 \\
\hline Rumination & & & & & & & & & & & & & 0.01 & 0.00 & 0.34 & $9.51^{* * * *}$ \\
\hline $\mathrm{R}^{2}$ & 0.076 & & & & 0.100 & & & & 0.133 & & & & 0.276 & & & \\
\hline $\operatorname{adj.} R^{2}$ & 0.075 & & & & 0.095 & & & & 0.128 & & & & 0.270 & & & \\
\hline F Statistic & $66.91^{* * * *}$ & $(d f=1$ & ;810) & & $22.45^{* * * *}$ & $(d f=4 ;$ & ;807) & & $24.88^{* * * *}$ & $(d f=5 ; 8$ & 306) & & $38.22^{* * *}$ & $(d f=8 ;$ & 303) & \\
\hline$\Delta \mathrm{R}^{2}$ & & & & & $0.024^{* * *}$ & & & & $0.033^{* * *}$ & & & & $0.143^{* * *}$ & & & \\
\hline
\end{tabular}

Note: $N=812 . B$ represents unstandardized regression coefficients; $S E$ represents the standard error of $B ; \beta$ represents standardised regression coefficients.

$* \mathrm{p}<0.05 ; * * \mathrm{p}<0.01 ; * * * \mathrm{p}<0.001$

\section{Discussion}

The objective of the present study was to advance understanding of online vigilance - a novel construct which describes individual differences in users' cognitive orientation to online connectedness, their attention to and integration of online-related cues and stimuli, and their prioritisation of online communication (Reinecke et al., 2018). Currently, only a small collection 
of studies have investigated online vigilance and theoretical frameworks describing its relationship with media use habits and personal characteristics are yet to be proposed. We aimed to take an initial step towards theorisation by investigating the degree to which particular media use patterns and personal characteristics explain its variance in a student population.

The results of our analysis indicate that, while it is a significant predictor, the amount of time users spend on their smartphones per day explains only $7.5 \%$ of variance in online vigilance. The weak effect of this predictor suggests that high degrees of online vigilance may be experienced by individuals despite low amounts of daily smartphone use. This finding provides support for the argument that care should be taken when high-level indicators like screentime are used as predictors of media effects (Orben \& Przybylski, 2019). Moreover, it calls into question the value of interventions like screentime tracking applications that focus primarily on users' monitoring of the overall time spent on their devices.

To address our second research question we adapted our model by adding variables indicating the frequency of respondents' use of three particular media types - social media, watching video content and messaging. Of these three, only social media was found to be a statistically significant predictor of online vigilance. It should be noted that, for some users, a degree of overlap may exist between social media use and messaging as the latter often occurs as an affordance of the former (e.g., instant messaging on Facebook). Nonetheless, we argue that, when considering users' cognitive orientation towards their online spheres, certain forms of media are more likely than others to engender vigilance. For example, we propose that the properties and affordances of social media, characterised by continuous social interactions which trigger frequent monitoring and responding, are more likely to produce highly vigilant users than online video platforms like Netflix. Accordingly, we propose that future studies of online vigilance elicit textured representations of a user's media use patterns (e.g., the types of and frequency with which particular forms of media and their affordances are used).

The addition of media multitasking as independent variable in the third step of the hierarchical regression, increased the model's explanatory power of variance in online vigilance by a further $3.3 \%$, but the model's overall effect remained weak. The standardised coefficients of the three significant predictors (daily smartphone use, social media, and media multitasking) indicate that, 
of these, media multitasking is the strongest predictor. This finding aligns with that of Reinecke et al. (2018) and supports their argument that media multitasking is indicative of the continuous prioritisation of online media use. However, while Reinecke et al. (2018, p. 14) report "particularly strong connections" between internet multitasking and online vigilance, our data are less convincing, showing only weak association between these constructs. This difference may be explained by our adoption of the MMI-S rather than the Internet multitasking scale used by Reinecke et al., (2018). While the MMI-S specifically measures multitasking between various media use activities, weighted by use time per activity, Reinecke et al.,'s instrument emphasises multitasking between online and offline activities.

In the final step of the hierarchical regression we added the three personal characteristics to the set of predictors. The updated model predicted $27 \%$ of variance in online vigilance, a significant increase of $14.3 \%$ from Model 3 which included only variables describing media use behaviour. Of the three personal characteristics, rumination emerged as the strongest predictor. This finding is indicative of the overlap between rumination and online vigilance which, it may be argued, can be framed as describing individuals' rumination about their online spheres. Interestingly, in Model 4, the standardised coefficient of identity distress is equal to that of media multitasking, indicating its value as a predictor of online vigilance. We argue, on this basis, that the reciprocal relationship between media use and identity distress is an important factor in online vigilance and should be considered in future studies. Lastly, it is worth noting that, within Model 4, emotional intelligence is not a significant predictor of online vigilance. Since our sample only included digital natives, this finding may be reflective of Hornung et al.'s (2018) proposition that emotional intelligence is developed along with social media use. We propose, accordingly, that emotional intelligence may be more relevant as a predictor of how students adopt and enact the different affordances of online platforms, as opposed to online vigilance.

We conclude by acknowledging several limitations to the present study. First, our cross-sectional design prevents any inferences about causal relations between the variables we used and online vigilance. For this, future work adopting either experimental or longitudinal methods would be required. Second, in using self-report measures to assess media use behaviour, our analyses are subject to the often poor accuracy of such reports (Ellis et al., 2019). Similarly, while the online 
vigilance scale has received some validation among European samples, the instrument has yet to be validated among a South African sample. Despite this, given similarities in media behaviour between these populations (le Roux and Parry, 2019b), while future validations should be conducted, we do not foresee substantial differences. As a final limitation, noting that online vigilance represents a compound construct (Reinecke et al., 2018), building on the present study, there is a need for future work to consider associations between various socio-emotional and behavioural factors and the three sub-components of online vigilance.

\section{References}

Baumgartner, S. E. et al. (2017) 'Measuring Media Multitasking: Development of a Short Measure of Media Multitasking for Adolescents', Journal of Media Psychology, 29(2), pp. 92101.

Berman, S. L., Montgomery, M. J. and Kurtines, W. M. (2004) 'The Development and Validation of a Measure of Identity Distress', Identity. Lawrence Erlbaum Associates, Inc., 4(1), pp. 1-8. doi: 10.1207/S1532706XID0401_1.

Brinker, J. K. and Dozois, D. J. A. (2009) 'Ruminative Thought Style and Depressed Mood', Journal of Clinical Psychology, 65(1), pp. 1-19.

Butler, L. D. and Nolen-Hoeksema, S. (1994) 'Gender differences in responses to depressed mood in a college sample', Sex Roles. Kluwer Academic Publishers-Plenum Publishers, 30(5-6), pp. 331-346. doi: 10.1007/BF01420597.

Dempsey, A. E. et al. (2019) 'Fear of missing out (FoMO) and rumination mediate relations between social anxiety and problematic Facebook use', Addictive Behaviors Reports. Elsevier, 9, p. 100150. doi: 10.1016/J.ABREP.2018.100150.

Ellis, D. A. et al. (2019) 'Do smartphone usage scales predict behavior?', International Journal of Human-Computer Studies. Elsevier, 130, pp. 86-92.

Hornung, O., Dittes, S. and Smolnik, S. (2018) 'When emotions go social-understanding the role of emotional intelligence in social network use', Proceedings of twenty-sixth European Conference on information Systems.

Johannes, N. et al. (2018) 'Mind-Wandering and Mindfulness as Mediators of the Relationship Between Online Vigilance and Well-Being', CyberPsychology, Behavior \& Social Networking, $00(00)$.

Kircaburun, K., Griffiths, M. D. and Billieux, J. (2019) 'Trait emotional intelligence and problematic online behaviors among adolescents: The mediating role of mindfulness, rumination, 
and depression', Personality and Individual Differences. Pergamon, 139, pp. 208-213. doi: 10.1016/J.PAID.2018.11.024.

le Roux, D. B. and Parry, D. A. (2019) 'Off-task media use in academic settings: cycles of selfregulation failure', Journal of American College Health, pp. 1-8. doi: 10.1080/07448481.2019.1656636.

le Roux, D. B. and Parry, D. A. (2019) 'Investigating differences in the attention distribution strategies of high and low media multitaskers through a two-dimensional game', Cyberpsychology: Journal of Psychosocial Research on Cyberspace, 13(3). doi: 10.5817/CP2019-3-2.

McLaughlin, K. A., Borkovec, T. D. and Sibrava, N. J. (2007) 'The Effects of Worry and Rumination on Affect States and Cognitive Activity', Behavior Therapy. Elsevier, 38(1), pp. 2338. doi: 10.1016/J.BETH.2006.03.003.

Nolen-Hoeksema, S. (2001) 'Gender Differences in Depression', Current Directions in Psychological Science. Sage: Los Angeles, CA, 10(5), pp. 173-176. doi: 10.1111/14678721.00142 .

Ophir, E., Nass, C. and Wagner, A. D. (2009) 'Cognitive control in media multitaskers.', Proceedings of the National Academy of Sciences of the United States of America, 106(37), pp. 15583-15587. doi: 10.1073/pnas.0903620106.

Orben, A. and Przybylski, A. K. (2019) 'The association between adolescent well-being and digital technology use', Nature Human Behaviour, 3(2), pp. 173-182. doi: 10.1038/s41562-0180506-1.

Petrides, K. V (2009) 'Psychometric Properties of the Trait Emotional Intelligence Questionnaire (TEIQue)', in Parker, J. D. A., Saklofske, D. H., and Stough, C. (eds) Assessing Emotional Intelligence: Theory, Research, and Applications. Boston, MA: Springer US, pp. 85-101. doi: 10.1007/978-0-387-88370-0_5.

Petrides, K. V., Pita, R. and Kokkinaki, F. (2007) 'The location of trait emotional intelligence in personality factor space', British Journal of Psychology, 98(2), pp. 273-289. doi: 10.1348/000712606X120618.

Przybylski, A. K. et al. (2013) 'Motivational, emotional, and behavioral correlates of fear of missing out', Computers in Human Behavior. Elsevier Ltd, 29(4), pp. 1841-1848. doi: 10.1016/j.chb.2013.02.014.

Reinecke, L. et al. (2018) 'Permanently online and permanently connected: Development and validation of the Online Vigilance Scale', Plos One, 13(10). doi: 10.1371/journal.pone.0205384. 
Schneider, F. M. and Hitzfeld, S. (2019) 'I Ought to Put Down That Phone but I Phub Nevertheless: Examining the Predictors of Phubbing Behavior', Social Science Computer Review, p. 089443931988236 . doi: 10.1177/0894439319882365.

Süral, I. et al. (2019) 'Trait Emotional Intelligence and Problematic Social Media Use Among Adults: The Mediating Role of Social Media Use Motives', International Journal of Mental Health and Addiction. Springer US, 17(2), pp. 336-345. doi: 10.1007/s11469-018-0022-6.

Ward, A. S. (2017) Adolescent Identity Formation and Social Media. Canterbury Christ Church University.

Wiradhany, W. and Nieuwenstein, M. (2017) 'Cognitive control in media multitaskers: Two replication studies and a meta-Analysis', Attention, Perception, \& Psychophysics. Attention, Perception, \& Psychophysics, pp. 1-22. doi: 10.3758/s13414-017-1408-4.

Yang, C. et al. (2018) 'Social media social comparison and identity distress at the college transition: A dual-path model', Journal of Adolescence. Academic Press, 69, pp. 92-102. doi: 10.1016/J.ADOLESCENCE.2018.09.007. 